\title{
Complement component 7 (C7), a potential tumor suppressor, is correlated with tumor progression and prognosis
}

\author{
Lisha Ying ${ }^{1,2, *}$, Fanrong Zhang ${ }^{2, *}$, Xiaodan Pan ${ }^{3}$, Kaiyan Chen ${ }^{2}$, Nan Zhang ${ }^{2}$, Jiaoyue \\ Jin'2, Junzhou Wu${ }^{2}$, Jianguo Feng ${ }^{2}$, Herbert $\mathbf{Y u}^{4}$, Hongchuan Jin ${ }^{1}$, Dan Su ${ }^{2}$ \\ ${ }^{1}$ Laboratory of Cancer Biology, Provincial Key Lab of Biotherapy in Zhejiang, Sir Runrun Shaw Hospital, Medical School of \\ Zhejiang University, Hangzhou, China \\ ${ }^{2}$ Cancer Research Institute, Zhejiang Cancer Hospital \& Key Laboratory Diagnosis and Treatment Technology on Thoracic \\ Oncology of Zhejiang Province, Hangzhou, China \\ ${ }^{3}$ Tissue Bank of Zhejiang Cancer Hospital, Hangzhou, China \\ ${ }^{4}$ Cancer Epidemiology Program, University of Hawaii Cancer Center, Hawaii, USA \\ *These authors have contributed equally to this work \\ Correspondence to: Dan Su, email: sudan@zjcc.org.cn \\ Hongchuan Jin, email: jinhc@zju.edu.cn \\ Keywords: complement component 7, tumor progression, prognosis, ovarian cancer, non-small cell lung cancer \\ Received: September 02, $2016 \quad$ Accepted: October 29, $2016 \quad$ Published: November 11, 2016
}

\section{ABSTRACT}

Our previous study found copy number variation of chromosome fragment 5p13.1-13.3 might involve in the progression of ovarian cancer. In the current study, the alteration was validated and complement component 7 (C7), located on 5p13.1, was identified. To further explore the clinical value of $C 7$ in tumors, 156 malignant, 22 borderline, 33 benign and 24 normal ovarian tissues, as well as 173 non-small cell lung cancer (NSCLC) tissues along with corresponding adjacent and normal tissues from the tissue bank of Zhejiang Cancer Hospital were collected. The expression of C7 was analyzed using reverse transcriptase quantitative polymerase chain reaction. As a result, the $\mathrm{C7}$ expression displayed a gradual downward trend in normal, benign, borderline and malignant ovarian tissues, and the decreased expression of $\mathrm{C7}$ was correlative to poor differentiation in patients with ovarian cancer. Interestingly, a similar change of expression of $\mathrm{C7}$ was found in normal, adjacent and malignant tissues in patients with NSCLC, and low expression of C7 was associated with worse grade and advanced clinical stage. Both results from this cohort and the public database indicated that NSCLC patients with low expression of C7 had a worse outcome. Furthermore, multivariate cox regression analysis showed NSCLC patients with low $\mathrm{C7}$ had a $\mathbf{3 . 0 9}$ or $\mathbf{5 . 6 5 - f o l d ~ h i g h e r ~ r i s k ~ f o r ~ r e l a p s e ~ o r ~ d e a t h ~ t h a n ~ t h o s e ~ w i t h ~}$ high $\mathrm{C7}$ respectively, suggesting $\mathrm{C7}$ was an independent prognostic predictor for prognoses of patients with NSCLC. Additionally, overexpression of C7 inhibited colony formation of NSCLC cells, which hints C7 might be a potential tumor suppressor.

\section{INTRODUCTION}

The complement system is a phylogenetically ancient makeup of the innate immune system and plays a vital role in immune surveillance and homeostasis [1, 2]. After activated through a linear cascade of separate pathways known as the classical, lectin and alternative pathways, complements trigger irreversible assembly of a multi-protein pore in cell membranes, the membrane attack complex (MAC) which functions as the cytolytic effector unit of complement system [1-3]. Apart from directly lysing malignant transformed cells, the complements cascade also enhances antibody-dependent cell-mediated cytotoxicity (ADCC) against tumor cells $[2,4]$.

The complement component 7 (C7) is a terminal component of the complement cascade and its insertion 
into the lipid bilayers is a critical limiting factor for the formation of MAC [5-7]. It is generally recognized the inherited deficiency of $\mathrm{C} 7$ is correlated with an increased susceptibility to a wide range of Gram-negative bacteria $[8,9]$. Although the majority of systemic C7 is derived from hepatic and marrow sources [10], an estimated thirty percent of $\mathrm{C} 7$ from local synthesis has been described also important in the formation of MAC [5, $6,10]$. However, the role of $\mathrm{C} 7$ synthesized in tumors was few studied.

In the current study, C7 was identified as a potential tumor suppressor in ovarian cancer and non-small cell lung cancer (NSCLC). Moreover, it is an independent predictor for patient outcome.

\section{RESULTS}

\section{Comparison of chromosome fragment 5p13.1- 13.3 between cell lines}

Our previous study [11] found that the high metastatic ovarian cancer cell line HO-8910PM had less copy number in chromosome fragment 5p13.1-13.3 than its parental cell line HO-8910. In this study, the fluorescent in situ hybridization (FISH) assay was carried out to verify this observation. By using specific biotin-labeled BAC probes which mapped into 5p13.1-13.3, one copy chromosomal hybridization signal was found in $\mathrm{HO}-$ 8910 (Figure 1A), while no signal was detected in HO-

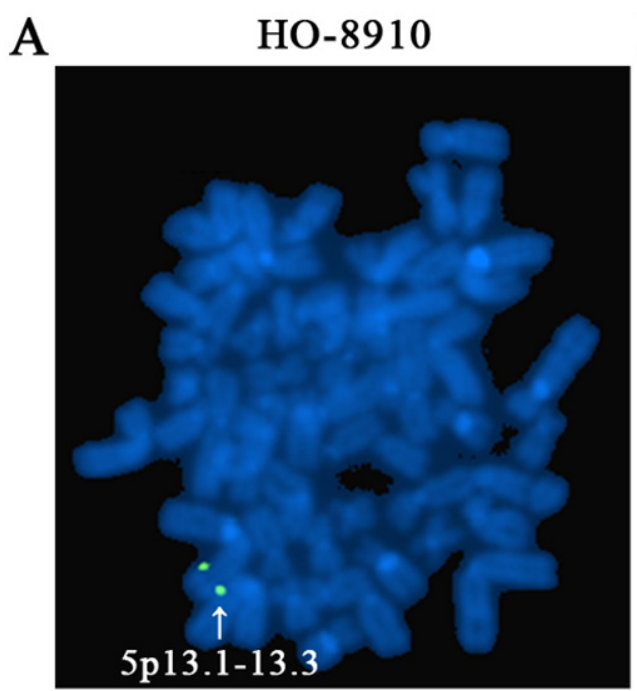

B

HO-8910PM

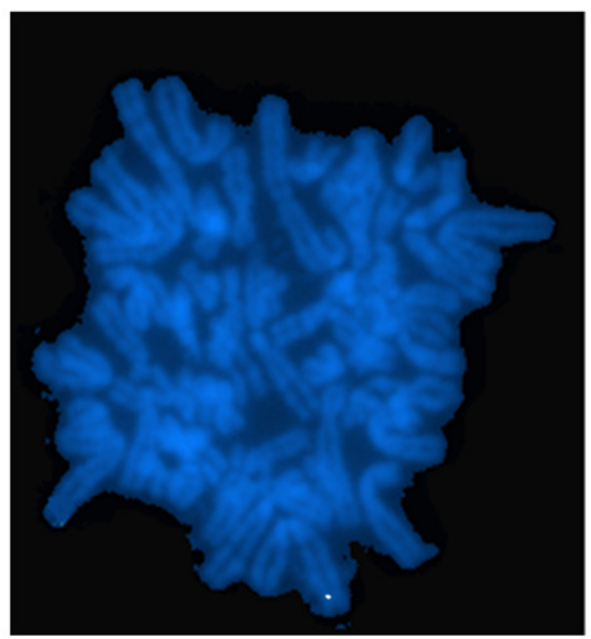

C

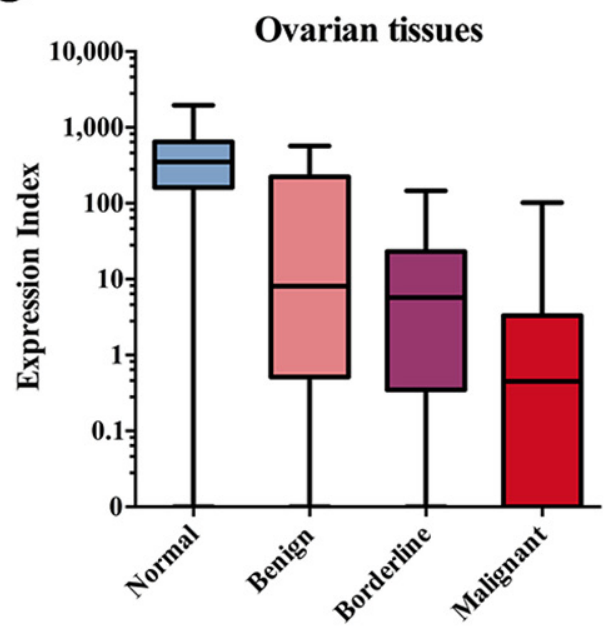

D

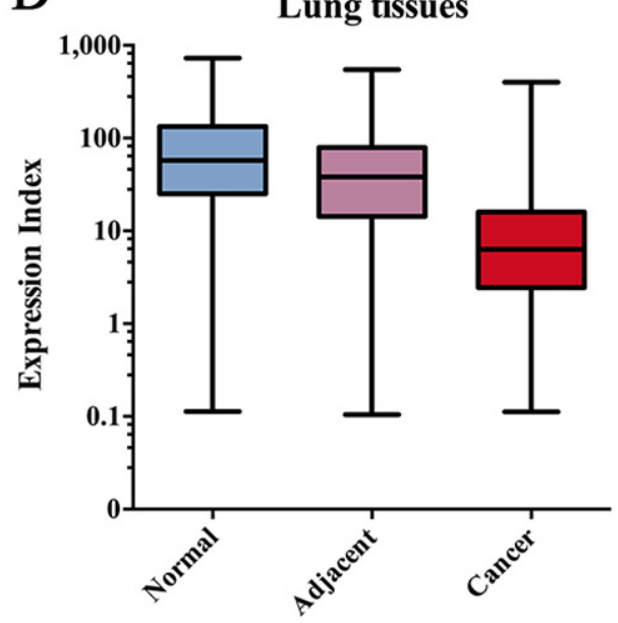

Figure 1: The deletion of C7 in tumor progression. A \& B. Detection of chromosome fragment 5p13.1-13.3 by FISH in HO-8910 and HO-8910PM. Green dots located on the sister chromatid were determined as real signals. Original magnification $\times 400$. C. Different C7 expression levels in ovarian tissues with diverse biological behaviors. The C7 mRNA expression of 4.2\% (1/24) normal tissues, $15.2 \%$ $(5 / 33)$ benign tissues, $13.6 \%$ (3/22) borderline tissues and 26.9\% (42/156) malignant tissues was too low to detect and thereby the $\mathrm{C} 7$ expression of those tissues was considered negative (Expression Index $=0$ ). Analyzed by the Kruskal-Wallis and the Dunn test, the C7 expression values were found significantly different between malignant and any other group. D. Different C7 expression levels in lung tissues with diverse biological behaviors. Significant difference of C7 expression was found between any two of lung groups. 
8910PM (Figure 1B), suggesting loss of heterozygosity of chromosome fragment 5p13.1-13.3 in HO-8910 and homozygous deletion of chromosome fragment $5 \mathrm{p} 13.1$ 13.3 in the parental cell line, which was identical with our previous CGH results. Combining with our previous microarray data on gene expression profiles [12] which showed that the $\mathrm{C} 7$ had the most significant decrease of expression among genes located on chromosome fragment 5p13.1-13.3 in HO-8910PM compared with HO-8910, the C7 was further studied.

\section{The clinical and prognostic values of $\mathrm{C} 7$ in ovarian cancer patients}

Firstly, the expression of $\mathrm{C} 7$ in ovarian tissues with diverse biological behaviors was analyzed. As a result, the mRNA expression levels of C7 in normal, benign, borderline and malignant ovarian tissues showed a gradual declining trend (Figure 1C). The median Expression Indexes of C7 expression were 351.13, 8.09, 5.77 and 0.45 in normal, benign, borderline and malignant ovarian tissues and significant difference of $\mathrm{C} 7$ expression was found between malignant and any other group.

Subsequently, the relation between $\mathrm{C} 7$ expression and clinicopathological factors, as well as its relation with outcomes in 156 ovarian cancer patients was investigated. As displayed in Table 1, both Mann-Whitney $\mathrm{U}$ test and chi-square test found that the expression of C7 was significantly related to tumor grade in patients with ovarian cancer. Patients with inferior tumor grade had a low expression level of C7. Nonetheless, the Cox regression model and Kaplan-Meier analysis didn't find any significant association between $\mathrm{C} 7$ expression and prognosis in patients with ovarian cancer (Table 2, Figures $2 \mathrm{~A}$ and $2 \mathrm{~B}$ ). The public database showed similar results (Figures 3A and 3B, http://kmplot.com/analysis/index. $\mathrm{php} ? \mathrm{p}=$ service \&cancer=ovar) [13].

\section{The role of $\mathrm{C} 7$ in NSCLC}

To further investigate the value of $\mathrm{C} 7$ in other types of cancer, a NSCLC cohort was studied. As displayed in Figure 1D, consistent downward trend of C7 expression following tumor progression was discovered in lung tissues. The median Expression Indexes of $\mathrm{C} 7$ expression were 57.49, 38.08 and 6.33 in paired normal, adjacent and malignant lung tissues and significant difference of C7 expression was found between any two groups.

As a consequence of correlation analyses, in addition to the tumor grade, the clinical stage was also found significantly associated with the $\mathrm{C} 7$ expression in patients with NSCLC (Table 3), and less C7 expression meant worse grade and advanced clinical stage. Notably, as presented in Table 2, the univariate Cox regression analysis implied that depressed $\mathrm{C} 7$ expression in tumor tissues was related to poor outcomes in NSCLC patients (crude HR 2.60, $95 \%$ CI 0.99-6.86, $P=0.053$ for middle level and PFS; crude HR 3.28, 95\% CI 1.27$8.46, P=0.014$ for low level and PFS; crude HR 4.22, 95\% CI 1.16-15.40, $P=0.029$ for middle level and OS; crude HR 6.95, 95\% CI 2.02-23.98, $P=0.002$ for low level and OS). Similar results were obtained from the Kaplan-Meier estimators (Figures 2C and 2D; $P=$ 0.033 for PFS; and $P=0.002$ for OS) and the public database (Figure 3C, http://kmplot.com/analysis/index. php? $p=$ service \&cancer=lung) [14]. What's more, the C7 mRNA expression in NSCLC tissues was identified to be an independent prognostic factor for both disease progression and death after adjusted by gender, age, smoking status, histology type, tumor grade and clinical stage. NSCLC patients with low- and middle-expression C7 would have worse PFS and OS contrasted to those with high-expression C7 (Table 2; adjust HR 1.68, 95\% CI $0.59-4.80, P=0.333$ for middle level and DFS; adjust HR 3.09, 95\% CI 1.09-8.71, $P=0.033$ for low level and DFS; adjust HR 2.56, $95 \%$ CI 0.63-10.35, $P=0.188$ for middle level and OS; adjust HR 5.65, $95 \%$ CI 1.4921.37, $P=0.011$ for low level and OS).

\section{C7 overexpression inhibited proliferation of NSCLC cells in vitro}

To assess the $\mathrm{C} 7$ expression level in vitro, a panel of established human NSCLC cell lines was gathered. As Figure 4A indicates, the $\mathrm{C} 7$ protein expression is negative in A549, NCI-H460, NCI-H1395 and low in SK-MES-1 compared with that in NCI-H1975 and U1752.

To further evaluate the effect of $\mathrm{C} 7$ on the growth of NSCLC cells, the C7-low expressed SK-MES-1 cells were stably transfected by $\mathrm{C} 7$ overexpression lentiviral vectors. As shown in Figure 4B, red fluorescence can be observed in almost all stable transfected cells. Furthermore, western bolt showed the objective $\mathrm{C} 7$ band in overexpressed SKMES-1 cells whereas none in control cells (Figure 4C), which confirmed the $\mathrm{C} 7$ overexpression in treated cells.

And then, the colony formation assay was done to explore the influence of C7 on proliferation ability of SKMES-1 cells. As Figures 4D and 4E illustrate, the colonies that the C7 overexpressed SK-MES-1 cells formed were nearly half of that control cells formed, which indicated that the C7 inhibited the growth of NSCLC cells in vitro.

\section{DISCUSSION}

Combining the result of FISH and our previous gene-expression profiling data [12], the C7 was identified. In the first place, the clinical and prognostic effects of $\mathrm{C} 7$ in ovarian cancer were investigated. As a result, the $\mathrm{C} 7 \mathrm{mRNA}$ content descended gradually in normal, benign, borderline and malignant ovarian tissues. And in patients with ovarian cancer, the decreased expression level of $\mathrm{C} 7$ was relative to worse 
Table 1: Association of C7 mRNA expression with clinicopathological factors in patients with ovarian cancer

\begin{tabular}{|c|c|c|c|c|c|c|c|}
\hline \multirow[b]{2}{*}{ Factors } & \multirow{2}{*}{$\begin{array}{l}\text { Case, } \\
\text { n (\%) }\end{array}$} & \multirow{2}{*}{$\begin{array}{c}\text { C7 expression } \\
\text { Median (5th-95th) }\end{array}$} & \multirow[b]{2}{*}{$P$ value } & \multicolumn{3}{|c|}{ C7 expression } & \multirow[b]{2}{*}{$P$ value } \\
\hline & & & & Low, n (\%) & Middle, n (\%) & High, n (\%) & \\
\hline Age (year) & & & 0.447 & & & & 0.199 \\
\hline$<60$ & $110(70.5)$ & $0.33(<0.01-21.29)$ & & $37(69.8)$ & $41(78.8)$ & $32(62.7)$ & \\
\hline$\geq 60$ & $46(29.5)$ & $0.84(<0.01-34.18)$ & & $16(30.2)$ & $11(21.2)$ & $19(37.3)$ & \\
\hline Histology & & & 0.630 & & & & 0.640 \\
\hline Non-serous & $42(26.9)$ & $0.79(<0.01-22.74)$ & & $12(22.6)$ & $16(30.8)$ & $14(27.5)$ & \\
\hline Serous & $114(73.1)$ & $0.36(<0.01-28.17)$ & & $41(77.4)$ & $36(69.2)$ & $37(72.5)$ & \\
\hline Grade & & & 0.011 & & & & 0.002 \\
\hline $1-2$ & $21(13.5)$ & $3.15(<0.01-71.95)$ & & $4(7.8)$ & $3(6.5)$ & $14(29.2)$ & \\
\hline 3 & $124(79.5)$ & $0.29(<0.01-15.76)$ & & $47(92.2)$ & $43(93.5)$ & $34(70.8)$ & \\
\hline Missing & $11(7.1)$ & & & & & & \\
\hline Clinical stage & & & 0.203 & & & & 0.062 \\
\hline I-II & $28(17.9)$ & $1.57(<0.01-30.51)$ & & $7(13.2)$ & $7(13.5)$ & $14(27.5)$ & \\
\hline III-IV & $128(82.1)$ & $0.39(<0.01-25.49)$ & & $46(86.8)$ & $45(86.5)$ & $37(72.5)$ & \\
\hline Residual Tumor & & & 0.165 & & & & 0.077 \\
\hline No residual & $78(50.0)$ & $0.67(<0.01-20.62)$ & & $32(61.5)$ & $20(39.2)$ & $26(51.0)$ & \\
\hline Residual & $76(48.7)$ & $0.73(<0.01-27.44)$ & & $20(38.5)$ & $31(60.8)$ & $25(49.0)$ & \\
\hline Missing & $2(1.3)$ & & & & & & \\
\hline
\end{tabular}

Bold values were statistically significant $(P \leq 0.05)$.

Table 2: Correlations between C7 mRNA expression and patient survival estimated by univariate and multivariate Cox regression analyses

\begin{tabular}{|c|c|c|c|c|c|c|c|}
\hline & C7 expression & Crude HR & $95 \% \mathrm{CI}$ & $P$ value & Adjust HR & $95 \%$ CI & $P$ value \\
\hline \multicolumn{8}{|l|}{ OC PFS } \\
\hline & High & 1 & & 0.287 & 1 & & 0.871 \\
\hline & Middle & 1.08 & $0.62-1.88$ & 0.782 & 1.05 & $0.58-1.90$ & 0.869 \\
\hline & Low & 0.71 & $0.40-1.27$ & 0.249 & 0.89 & $0.48-1.68$ & 0.727 \\
\hline \multicolumn{8}{|l|}{ OC OS } \\
\hline & High & 1 & & 0.326 & 1 & & 0.656 \\
\hline & Middle & 1.17 & $0.64-2.13$ & 0.618 & 1.36 & $0.70-2.64$ & 0.368 \\
\hline & Low & 0.74 & $0.40-1.36$ & 0.329 & 1.10 & $0.56-2.17$ & 0.782 \\
\hline \multicolumn{8}{|l|}{ NSCLC PFS } \\
\hline & High & 1 & & 0.047 & 1 & & 0.087 \\
\hline & Middle & 2.60 & $0.99-6.86$ & 0.053 & 1.68 & $0.59-4.80$ & 0.333 \\
\hline & Low & 3.28 & $1.27-8.46$ & 0.014 & 3.09 & $1.09-8.71$ & 0.033 \\
\hline \multicolumn{8}{|l|}{ NSCLC OS } \\
\hline & High & 1 & & 0.008 & 1 & & 0.025 \\
\hline & Middle & 4.22 & $1.16-15.40$ & 0.029 & 2.56 & $0.63-10.35$ & 0.188 \\
\hline & Low & 6.95 & $2.02-23.98$ & 0.002 & 5.65 & $1.49-21.37$ & 0.011 \\
\hline
\end{tabular}

Bold values were statistically significant $(P \leq 0.05)$.

$H R$ hazard ratio, CI confidence interval, $P F S$ progression free survival, $O S$ overall survival, $O C$ ovarian cancer, NSCLC non-small cell lung cancer 
grade. However, it is failing to find any prognostic value of C7 in ovarian cancer from this study and the public database. To further explore the clinical significance of $\mathrm{C} 7$ in other malignancies, a number of patients with NSCLC were gathered and consistent downward tendency of C7 mRNA expression was found in the course of lung tumor progression. Apart from tumor grade, clinical stage was also detected correlative to the expression level of C7 in NSCLC patients. And

A

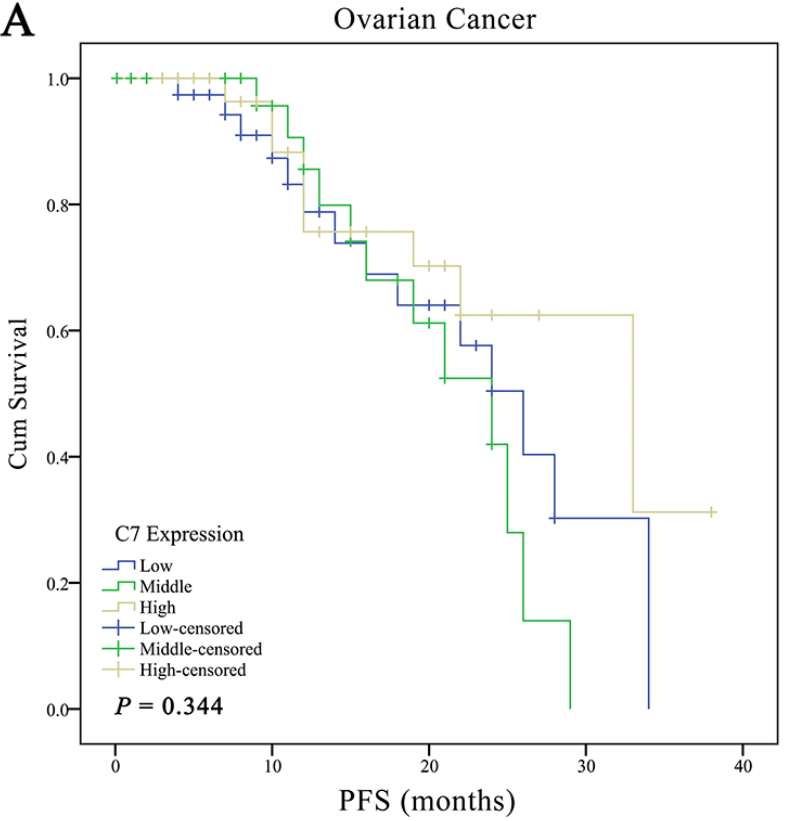

$\mathrm{C}$

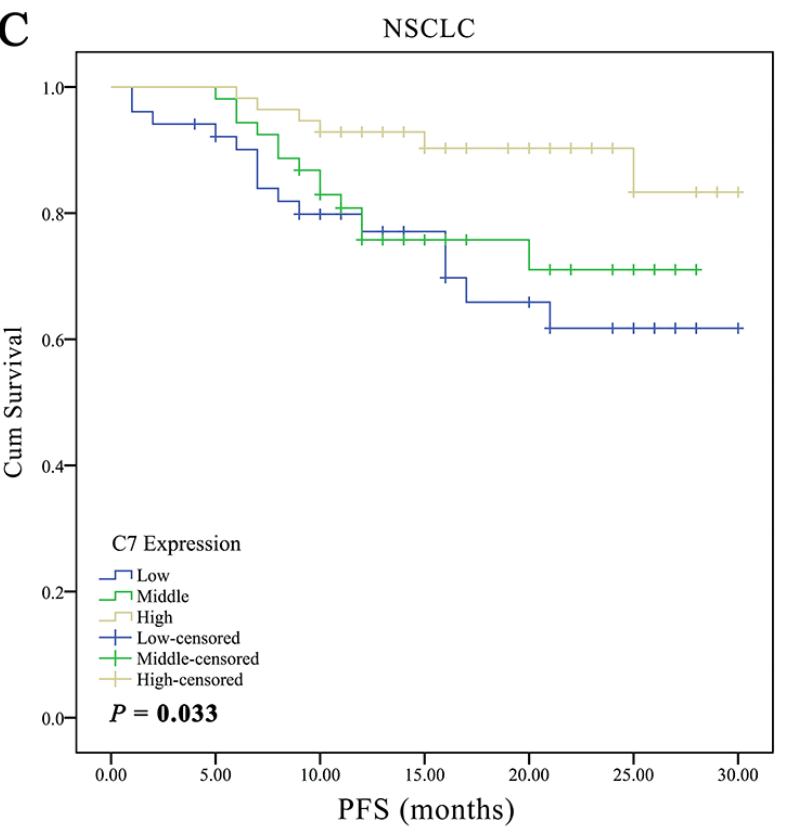

both results from this cohort and the public database demonstrated that NSCLC patients with decreased expression of $\mathrm{C} 7$ had a worse outcome. Moreover, the C7 mRNA expression level in cancer tissues was an independent predictor for both disease progression and death in patients with NSCLC. It is speculated from the foregoing that the $\mathrm{C} 7$ is a potential tumor suppressor and may make more contributions to NSCLC than ovarian cancer.
B

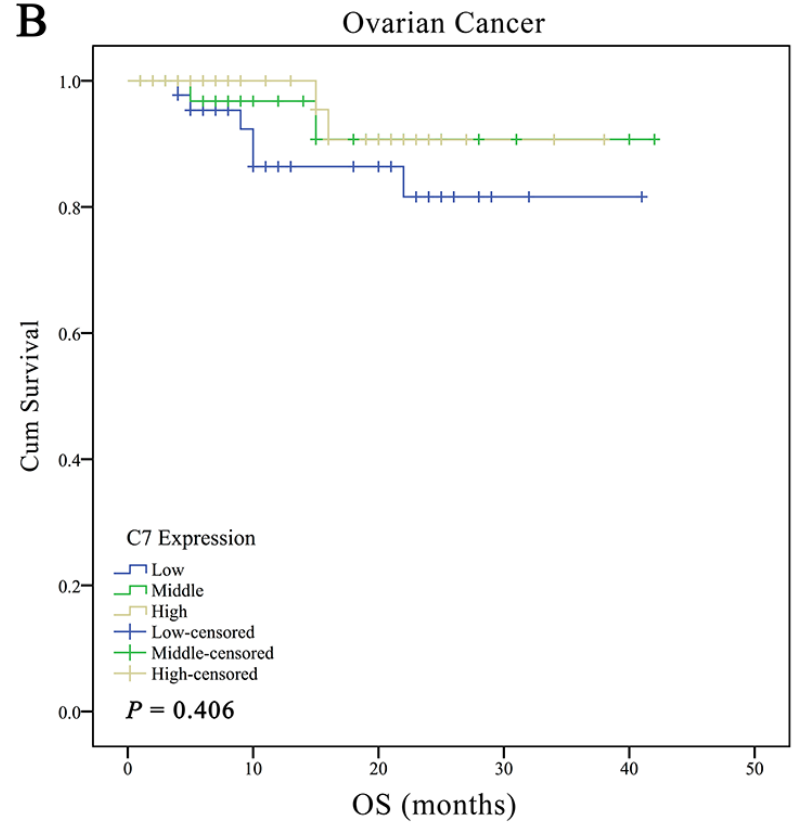

D

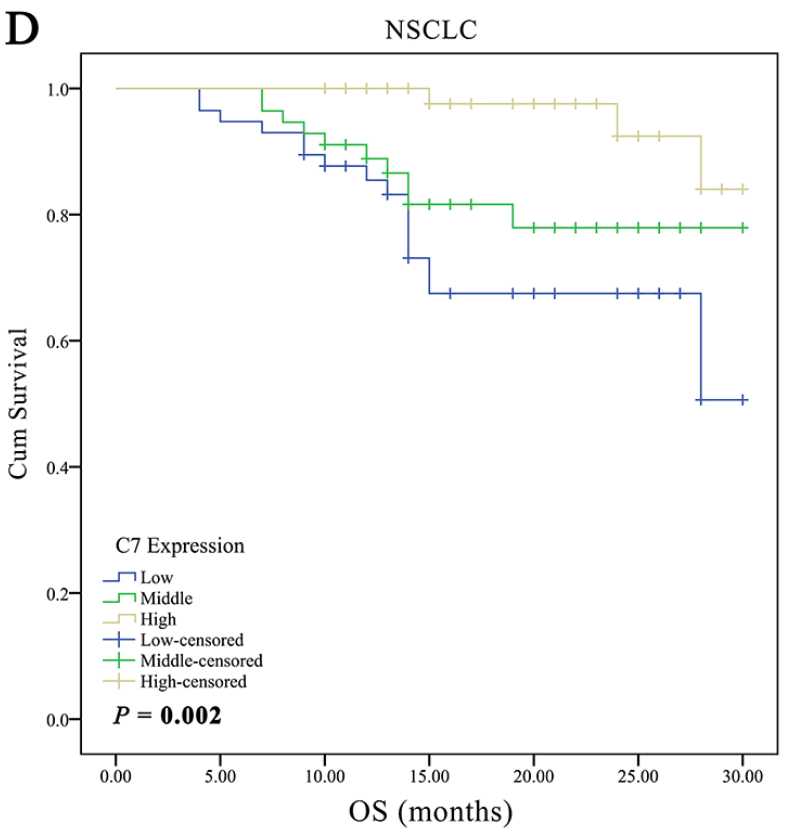

Figure 2: Kaplan-Meier curves according to the $\mathrm{C} 7$ expression levels for progression free survival and overall survival of patients with ovarian cancer or non-small cell lung cancer. The survival curves were developed using Kaplan-Meier estimator and the $P$ value was calculated through Log-rank test. $P F S$ progression free survival, $O S$ overall survival, NSCLC non-small cell lung cancer. 

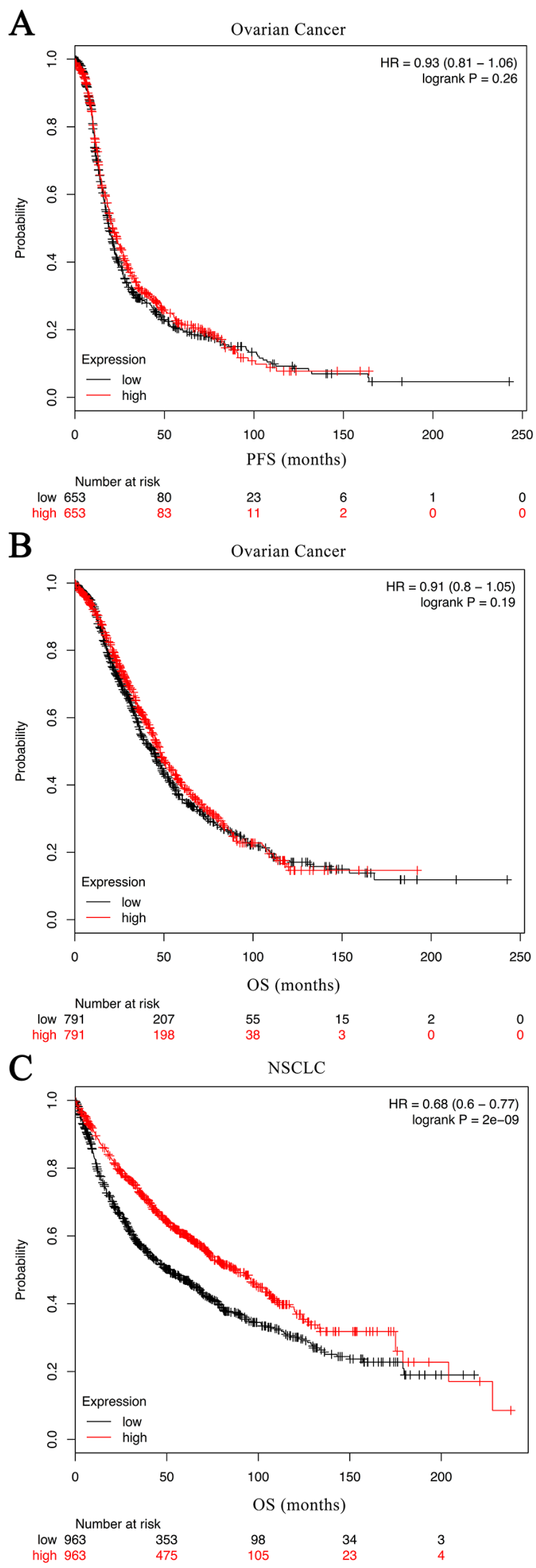

Figure 3: Kaplan-Meier curves created by the public database and web application KM plotter (http://kmplot.com/ analysis/) based on the C7 expression levels. Analysis for PFS of NSCLC was unavailable in this database. HR hazard ratio, PFS progression free survival, $O S$ overall survival, NSCLC non-small cell lung cancer. 
Table 3: Association of C7 mRNA expression with clinicopathological factors in patients with non-small cell lung cancer

\begin{tabular}{|c|c|c|c|c|c|c|c|}
\hline \multirow[b]{2}{*}{ Factors } & \multirow[b]{2}{*}{ Case,n (\%) } & \multirow{2}{*}{$\begin{array}{c}\text { C7 expression } \\
\text { Median (5th-95th) }\end{array}$} & \multirow[b]{2}{*}{$P$ value } & \multicolumn{3}{|c|}{ C7 expression } & \multirow[b]{2}{*}{$P$ value } \\
\hline & & & & Low, n (\%) & Middle, n (\%) & High, n (\%) & \\
\hline Gender & & & 0.653 & & & & 0.954 \\
\hline Male & $141(81.5)$ & $5.82(0.54-166.46)$ & & $46(80.7)$ & $48(82.8)$ & $47(81.0)$ & \\
\hline Female & $32(18.5)$ & $7.32(0.17-145.71)$ & & $11(19.3)$ & $10(17.2)$ & $11(19.0)$ & \\
\hline Age (year) & & & 0.544 & & & & 0.825 \\
\hline$<65$ & $124(71.7)$ & $5.85(0.33-106.07)$ & & $41(71.9)$ & $40(69.0)$ & $43(74.1)$ & \\
\hline$\geq 65$ & $49(28.3)$ & $7.53(0.90-264.45)$ & & $16(28.1)$ & $18(31.0)$ & $15(25.9)$ & \\
\hline Smoking & & & 0.714 & & & & 0.724 \\
\hline Never & $36(20.8)$ & $6.29(0.38-97.00)$ & & $14(26.4)$ & $11(20.8)$ & $11(20.8)$ & \\
\hline Ever/current & $123(71.1)$ & $5.83(0.37-214.44)$ & & $39(73.6)$ & $42(79.2)$ & $42(79.2)$ & \\
\hline Missing & $14(8.1)$ & & & & & & \\
\hline Histology & & & 0.617 & & & & 0.605 \\
\hline SCC & $96(55.5)$ & $5.55(0.53-128.19)$ & & $36(63.2)$ & $29(50.0)$ & $31(53.4)$ & \\
\hline Adenocarcinoma & $66(38.2)$ & $7.31(0.45-144.01)$ & & $17(29.8)$ & $25(43.1)$ & $24(41.4)$ & \\
\hline Others & $11(6.4)$ & $3.44(0.20-38.95)$ & & $4(7.0)$ & $4(6.9)$ & $3(5.2)$ & \\
\hline Grade & & & 0.031 & & & & 0.157 \\
\hline High & $84(48.6)$ & $7.82(0.90-152.18)$ & & $22(41.5)$ & $29(55.8)$ & $33(58.9)$ & \\
\hline Low & $77(44.5)$ & $4.05(0.33-307.61)$ & & $31(58.5)$ & $23(44.2)$ & $23(41.1)$ & \\
\hline Missing & $12(6.9)$ & & & & & & \\
\hline Clinical stage & & & $<0.001$ & & & & $<0.001$ \\
\hline I-II & $118(68.2)$ & $8.03(0.56-91.76)$ & & $30(52.6)$ & $38(65.5)$ & $50(86.2)$ & \\
\hline III-IV & $55(31.8)$ & $3.26(0.21-350.76)$ & & $27(47.4)$ & $20(34.5)$ & $8(13.8)$ & \\
\hline
\end{tabular}

Bold values were statistically significant $(P \leq 0.05)$.

$S C C$ squamous cell carcinoma

Being the central protein of terminal complement cascade, the $\mathrm{C} 7$ is an integral part in the formation of MAC and the activation of host defence specifically to the inflammatory region $[5,6,10]$. Besides, the $\mathrm{C} 7$ expressed on the cell membrane was reported to have an additional role acting as a regulator of the excessive proinflammatory reaction [15]. Therefore, it is concluded that the reduction of $\mathrm{C} 7$ expression in tumors prevents the lysis of abnormal cells, enhances the resistance of cancer cells against complement attack, aggravates the inflammation around tumors and finally promotes the malignant progression.

Also, the present study demonstrated that the $\mathrm{C} 7$ overexpression suppressed the growth of NSCLC cells in vitro. The underlying mechanism hadn't been studied before. It was reported that the sublytic MACs (SC5b-C9) which forms when nucleated cells are not unequivocally identified as non-self $[5,15]$ can activate a few oncogenic pathways including the mitogen-activated protein kinase (MAPK) family, extracellular regulated protein kinases (ERKs), p38 MAPK, the phosphatidylinositol 3-kinase (PI3K) pathway, Ras [16] and also inhibit apoptosis by causing the phosphorylation of Bad and preventing the activation of Bid, FLIP and caspase-8 [17]. These discoveries provide clues for further understanding of the growth promoting impact of $\mathrm{C} 7$.

Identified with this study, Oka et al. found that the C7 expression was noticeably reduced in oesophageal carcinoma with paired normal specimens as control [18]. On the contrary, Suryawanshi et al. observed that the C7 in tumor-related endometriosis had higher expression levels than in normal endometrium [19]. The further 
reasons underlying the difference need to be explored. Some studies implied that a few complements such as $\mathrm{C} 3 \mathrm{a}$ and $\mathrm{C} 5 \mathrm{a}$ also contributed to cancer progression through diverse mechanisms including modulating the inflammatory response in the vicinity of tumor and creating favorable microenvironments for tumor development [20-22].

In summary, the present study showed for the first time that the $\mathrm{C} 7$ acted as a tumor suppressor, and its expression level was a potential prognostic predictor for both disease progression and death in patients with NSCLC. However, controversy existed that the C7 was identified by integrated genomics while its expression was measured in a RNA level in this study. As a matter of fact, we couldn't find an appropriate kit or approach to detect the copy number variations of $\mathrm{C} 7$. In addition, the representativeness of our sample was limited since tissues used in this study were from a single institution. As such, the results remain to be validated, and also, the molecular mechanisms need to further elucidate.

A

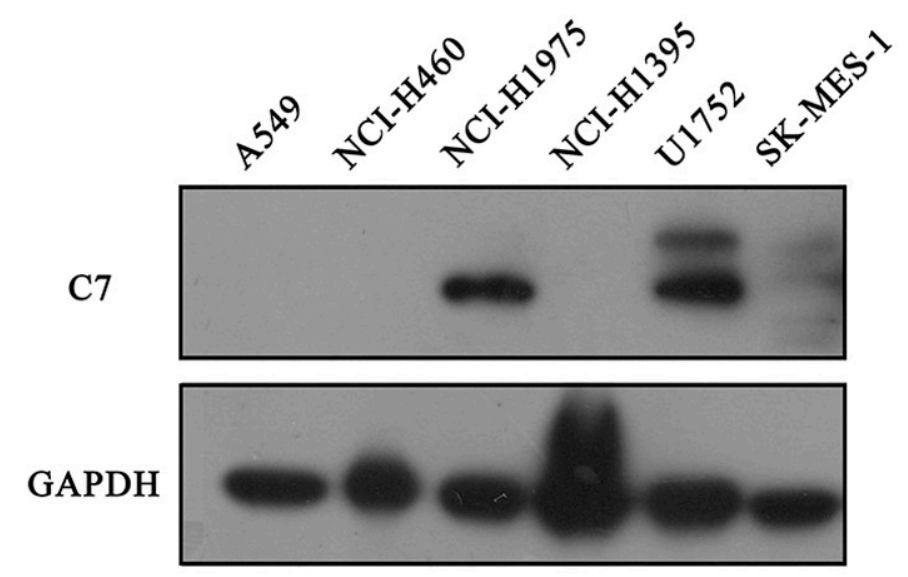

B
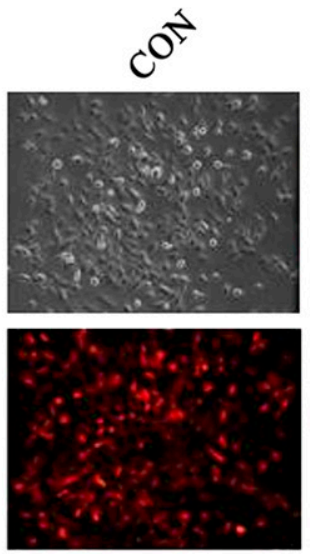

D

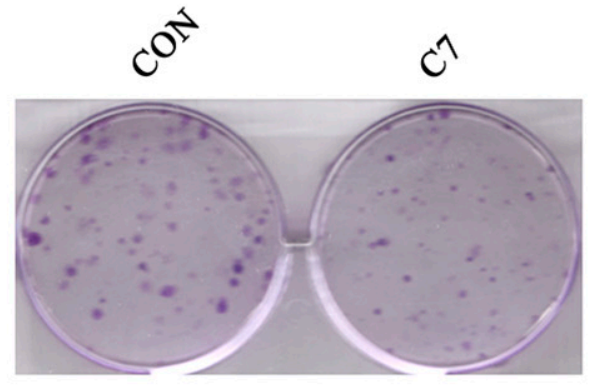

$\mathrm{C}$
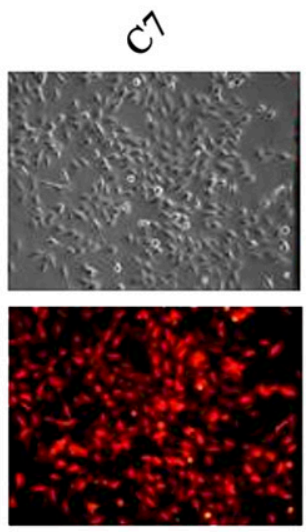

$\mathrm{E}$

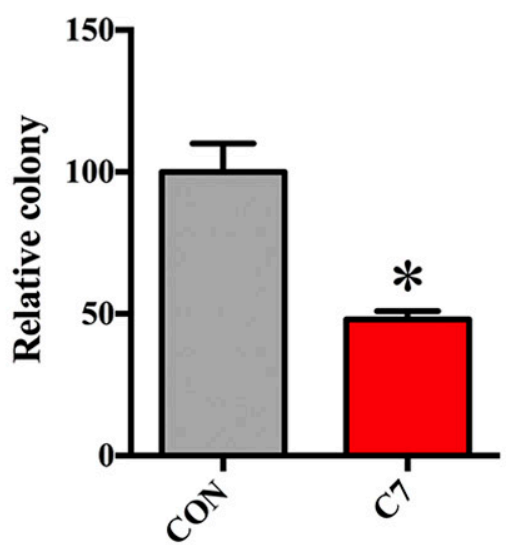

Figure 4: C7 overexpression significantly depressed the proliferation of SK-MES-1 cells. A. Expression of C7 protein in NSCLC cell lines. B. Red fluorescence of SK-MES-1 cells infected by lentiviral vectors. C. C7 protein levels examined by western blot. D. Colony formation assay of stable SK-MES-1 cells. E. Histogram of colony formation assay. The Student's t-test was used to evaluate the statistical significance. ${ }^{*}, P<0.05$. 


\section{MATERIALS AND METHODS}

\section{Cell culture}

Human ovarian cancer cell line HO-8910 and its derivative HO-8910PM with high metastatic capability were established in our previous studies [23, 24]; human NSCLC cell lines A549, NCI-H460, NCI-H1975, NCI-H1395, U1752 and SK-MES-1 were purchased from the Cell Bank of the Chinese Academy of Sciences, Shanghai, China. SK-MES-1 cells were cultured in Minimum Essential Medium (MEM, Gibco) supplemented with $10 \%$ fetal bovine serum (FBS, Gibco); other cells were cultured in RPMI 1640 medium (Gibco) supplemented with $10 \% \mathrm{FBS}$. All cells were maintained in a $37{ }^{\circ} \mathrm{C}, 5 \% \mathrm{CO}_{2}$ incubator.

\section{FISH}

This assay was performed as previously described [11]. Briefly, chromosomes from HO-8910 and HO8910PM were prepared with colchicine. Biotin labeled bacterial artificial chromosome (BAC) probes which specifically mapped onto 5p13.1-13.3 (SinoGenoMax Co., Ltd., Beijing, China) were applied to hybridize to the fixed cell nuclei. The signal of hybridization was enlarged through avidin-FITC and observed using a fluorescence microscope (Nikon, Tokyo, Japan) with FITC filter. Images were taken at a magnication of 400 $\times$. Chromosomes extracted from normal peripheral blood lymphocytes were used as control.

\section{Tissues and patients}

\section{Tissues}

One hundred and fifty-six malignant ovarian tissues from patients with ovarian cancer, 22 borderline, 33 benign and 24 normal ovarian tissues as well as 173 NSCLC tissues along with corresponding adjacent and normal tissues from patients with NSCLC were applied from the tissue bank of Zhejiang Cancer Hospital. All patients underwent surgery by experienced surgeons of Zhejiang Cancer Hospital and had provided informed consent before operation. This study was approved by the Institutional Review Board of Zhejiang Cancer Hospital.

\section{Patients with ovarian cancer}

A total of 156 patients with ovarian cancer treated in Zhejiang Cancer Hospital were retrospectively studied. As presented in Table 1, $70.5 \%(110 / 156)$ were younger than 60 years, $48.7 \%(76 / 156)$ had residual tumors after surgeries. According to the histological typing of ovarian tumors recommended by World Health Organization (WHO) [25], $73.1 \%(114 / 156)$ were serous tumor, and $26.9 \%(42 / 156)$ were non-serous tumor. As for tumor grade, $13.5 \%(21 / 156)$ were well or moderately differentiated (grade 1-2), $79.5 \%$ (124/156) were poorly differentiated (grade 3), and this information was missing in 11 cases $(7.1 \%)$. In accordance with the standard of International Federation of Gynecology and Obstetrics (FIGO) [26], 28 (17.9\%) patients were stage I-II, 128 $(82.1 \%)$ patients were stage III-IV.

Follow-up proceeded every three months for the first postoperative year and every six months for the subsequent years until patients died or lost. A routine follow-up evaluation included inquiry, physical examination, blood test, gynecological ultrasoundgraphy. The follow-up data comprised time of follow-up, time of relapse or metastasis, metastatic site, progression free survival (PFS), time of death, causa mortis and overall survival (OS). The median follow-up time was 40 (range 4-75) months. During the follow-up, $51.3 \%(80 / 156)$ patients progressed, $32.7 \%$ $(51 / 156)$ died and $9.0 \%(14 / 156)$ lost.

\section{Patients with NSCLC}

A total of 173 patients with NSCLC who underwent surgical treatment in Zhejiang Cancer Hospital were collected and their clinicopathological characteristics are summarized in Table 3. Among of them, $81.5 \%$ (141/173) were male, $71.7 \%(124 / 173)$ aged less than 65 years old, $71.1 \%(123 / 173)$ had smoking experience. On the basis of the WHO classification criteria [27], $55.5 \%(96 / 173)$ were squamous cell carcinoma, $38.2 \%(66 / 173)$ were adenocarcinoma, and $6.4 \%(11 / 173)$ were others. Of the 161 patients with data of tumor grade, 84 patients were high-grade, whereas 77 were low-grade. Based on the TNM classification of IASLC [28], 118 (68.2\%) patients were stage I-II, 55 (31.8\%) patients were stage III-IV.

The median follow-up time of patients with NSCLC was 15 (range 4-30) months. During the follow-up, 23.1 $\%(40 / 173)$ patients progressed, $16.8 \%(29 / 173)$ died and $1.2 \%(2 / 173)$ lost.

\section{Reverse transcriptase quantitative polymerase chain reaction (RT-qPCR)}

Total RNA was isolated from tissues using RNeasy Mini kits (QIAGEN Inc., Valencia, CA, USA). Five hundred nanograms of RNA were used for reverse transcription using PrimeScript ${ }^{\mathrm{TM}}$ RT Reagent Kit (Takara Biotechnology Co., Ltd., Dalian, China). Quantitative PCR was performed to determine gene expression level on ABI 7500 real-time PCR system (Applied Biosystems Inc., Foster City, CA, USA) using SYBR ${ }^{\circledR}$ Premix Ex Taq $^{\mathrm{TM}}$ II (Takara Biotechnology). The sequences of primers were: TGGAAACCCAGTGGCCAGA (C7 forward), GCCATCCATCAGTACAGGTAGAACA (C7 reverse), GAAGGTGAAGGTCGGAGTC (GAPDH forward), GAAGATGGTGATGGGATTTC (GAPDH reverse). The qPCR was carried out under the following conditions: $50^{\circ} \mathrm{C}$ for 2 minutes, $95^{\circ} \mathrm{C}$ for 10 minutes, 
40 cycles of $95^{\circ} \mathrm{C}$ for 15 seconds followed by $60^{\circ} \mathrm{C}$ for 1 minute, and a standard dissociation stage. The results of qPCR were analyzed using the software SDS 2.2.2 (Applied Biosystems) and recorded as threshold cycle $\left(C_{T}\right)$ values. The expression levels of target gene $\mathrm{C} 7$ were adjusted by housekeeping gene GAPDH and compared using the Expression Index $\left(2^{-\Delta \mathrm{CT}}\right)$ in accordance with our previous study [11].

\section{Western blot}

A549, NCI-H460, NCI-H1975, NCI-H1395, U1752 and SK-MES-1 cells were harvested by scraping, rinsed three times in phosphate-buffered saline (PBS), and lysed in NP40 cell lysis buffer supplemented with protease inhibitor cocktails. The protein concentration of the cell lysate was measured with the Bradford calorimetric assay (Bio-Rad, Richmond, CA, USA). Thirty micrograms of total protein were electrophoresed on an $8 \%$ SDSpolyacrylamide gel electrophoresis (SDS-PAGE) gel, and the separated proteins on the gel were transferred onto a polyvinylidene fluoride membrane (PVDF, Millipore, Bedford, MA, USA). Then the membrane was blocked with TBS-Tween-20 (TBS-T) containing 5\% non-fat milk for 2 hours at room temperature, followed by incubation with anti-C7 antibody (Epitomics Inc., Burlingame, CA, USA) overnight at $4^{\circ} \mathrm{C}$. Afterwards the membrane was incubated with a HRP-conjugated secondary antibody and detected with enhanced chemiluminescence regent (ECL, Millipore). GAPDH (Sigma, St Louis, MO, USA) was used as a loading control.

\section{C7 overexpression and colony formation assay}

The C7 overexpression lentiviral vector, pLenti-U6EF1 $\alpha$-C7-FLAG-P2A-tdTomato-T2A-Puromycin, and the empty lentiviral vector for control were purchased from 3D High Throughput Screening Co., Ltd. The lentiviral vectors were co-transfected with helper plasmids Gag pol, VSVG, REV (Invitrogen, Carlsbad, CA, USA) into 293T cells. Then the supernatant was harvested at 48 hours post transfection and purified by centrifugation and filtration using Millex-HV Syringe Filter Units with a $0.45 \mu \mathrm{m}$ pore size hydrophilic PVDF membrane (Millipore).

SK-MES-1 cells at 40\% - 50\% confluence were infected overnight with $\mathrm{C} 7$ overexpression or control lentiviral in $8 \mathrm{ug} / \mathrm{ml}$ polybrene. Stable SK-MES-1 cells were selected with $2 \mathrm{ug} / \mathrm{ml}$ puromycin for 2 weeks followed by maintenance in $1 \mathrm{ug} / \mathrm{ml}$ puromycin. Then the $\mathrm{C} 7$ overexpression in stable SK-MES-1 cells was validated by western bolt.

A total of 2,000 stable SK-MES-1 cells in $2 \mathrm{ml}$ complete medium were seeded onto 6-well plates. After incubating for 2 weeks, cells colonies were fixed with $4 \%$ paraformaldehyde, stained with $0.1 \%$ crystal violet and then colonies containing more than 50 cells were counted. The assay was replicated thrice in triplicate and the
Student's t-test was performed to calculate the statistical significance.

\section{Statistical analysis}

Data of C7 mRNA expression from patients' tissues were analyzed both as continuous and categorical variables. For categorical analysis, the continuous data of C7 expression were divided into low, middle and high groups according to tertile distributions. The difference of C7 expression among tissues with diverse biological behaviors was analyzed using Kruskal-Wallis with Dunn multiple comparisons test. The associations between $\mathrm{C} 7$ expression and clinicopathological factors were examined using Mann-Whitney $U$ test and chi-square test. The survival curves based on the C7 expression levels were generated by Kaplan-Meier estimator and the statistical difference was calculated through Log-rank test. A Cox proportional hazards regression model was performed to acquire the prognostic value of the $\mathrm{C} 7$ expression. The public database and web application named KM plotter (http://kmplot.com/analysis/) was used to verify the prognostic results [29]. SPSS 21.0 for Windows (IBM) was used for data analyses, and a $P$ value $\leq 0.05$ in a twotailed test was deemed of statistical significance.

\section{ACKNOWLEDGMENTS}

This study was supported by grants from the National Nature Science Foundation of China (No. 81472203), the Major Science and Technology Project of Medical and Health of Zhejiang Province of China (No. WKJ-ZJ-1403), the Major Science and Technology Project of Zhejiang Province of China (No. 2014C03029), the Natural Science Foundation of Zhejiang Province (No. LY13H160028), the Zhejiang Provincial Medicine and Health Science Fund (Nos. 2013KYA028, 2015KYA035), and the 1022 program of Zhejiang Cancer Hospital.

\section{CONFLICTS OF INTEREST}

The authors declare that they have no conflicts of interest.

\section{REFERENCES}

1. Ricklin D, Hajishengallis G, Yang K, Lambris JD. Complement: a key system for immune surveillance and homeostasis. Nat Immunol. 2010; 11: 785-97. doi: 10.1038/ ni.1923.

2. Serna M, Giles JL, Morgan BP, Bubeck D. Structural basis of complement membrane attack complex formation. Nat Commun. 2016; 7: 10587. doi: 10.1038/ncomms10587.

3. Podack ER. Molecular composition of the tubular structure of the membrane attack complex of complement. J Biol Chem. 1984; 259: 8641-7. 
4. Gelderman KA, Tomlinson S, Ross GD, Gorter A. Complement function in mAb-mediated cancer immunotherapy. Trends Immunol. 2004; 25: 158-64. doi: 10.1016/j.it.2004.01.008.

5. Wurzner R. Modulation of complement membrane attack by local C7 synthesis. Clin Exp Immunol. 2000; 121: 8-10.

6. Wurzner R, Joysey VC, Lachmann PJ. Complement component C7. Assessment of in vivo synthesis after liver transplantation reveals that hepatocytes do not synthesize the majority of human C7. J Immunol. 1994; 152: 4624-9.

7. Shen YB, Zhang JB, Fu JJ, Xu XY, Li JL, Wang RQ, Xuan YF. A $425 \mathrm{~T}>\mathrm{C}$ polymorphism in complement $\mathrm{C} 7$ association with resistance to Aeromonas hydrophila in grass carp. Genet Mol Res. 2016; 15. doi: 10.4238/ gmr.15014526.

8. Botto M, Kirschfink M, Macor P, Pickering MC, Wurzner $\mathrm{R}$, Tedesco F. Complement in human diseases: Lessons from complement deficiencies. Mol Immunol. 2009; 46: 2774-83. doi: 10.1016/j.molimm.2009.04.029.

9. Morgan BP, Walport MJ. Complement deficiency and disease. Immunol Today. 1991; 12: 301-6. doi: 10.1016/0167-5699(91)90003-c.

10. Naughton MA, Walport MJ, Wurzner R, Carter MJ, Alexander GJ, Goldman JM, Botto M. Organ-specific contribution to circulating $\mathrm{C} 7$ levels by the bone marrow and liver in humans. Eur J Immunol. 1996; 26: 2108-12. doi: 10.1002/eji.1830260922.

11. Su D, Katsaros D, Xu S, Xu H, Gao Y, Biglia N, Feng J, Ying L, Zhang P, Benedetto C, Yu H. ADP-ribosylation factor-like 4C (ARL4C), a novel ovarian cancer metastasis suppressor, identified by integrated genomics. Am J Transl Res. 2015; 7: 242-56.

12. Xu SH, Mu HZ, Gu LH, Zhu CH, Liu XL. Identification of differentially expressed genes in the high and low metastatic human ovarian cancer cell lines and analyses of their chromosomal localizations and functions. Yi Chuan Xue Bao. 2006; 33: 892-900. doi: 10.1016/ s0379-4172(06)60123-6.

13. Gyorffy B, Lanczky A, Szallasi Z. Implementing an online tool for genome-wide validation of survival-associated biomarkers in ovarian-cancer using microarray data from 1287 patients. Endocr Relat Cancer. 2012; 19: 197-208. doi: 10.1530/ERC-11-0329.

14. Chellappan SP, Győrffy B, Surowiak P, Budczies J, Lánczky A. Online Survival Analysis Software to Assess the Prognostic Value of Biomarkers Using Transcriptomic Data in Non-Small-Cell Lung Cancer. PLoS ONE. 2013; 8: e82241. doi: 10.1371/journal.pone.0082241.

15. Bossi F, Rizzi L, Bulla R, Debeus A, Tripodo C, Picotti P, Betto E, Macor P, Pucillo C, Wurzner R, Tedesco F. $\mathrm{C} 7$ is expressed on endothelial cells as a trap for the assembling terminal complement complex and may exert anti-inflammatory function. Blood. 2009; 113: 3640-8. doi: 10.1182/blood-2008-03-146472.
16. Pio R, Corrales L, Lambris JD. The role of complement in tumor growth. Adv Exp Med Biol. 2014; 772: 229-62. doi: 10.1007/978-1-4614-5915-6_11.

17. Tegla CA, Cudrici C, Patel S, Trippe R, 3rd, Rus V, Niculescu F, Rus H. Membrane attack by complement: the assembly and biology of terminal complement complexes. Immunol Res. 2011; 51: 45-60. doi: 10.1007/s12026-011-8239-5.

18. Oka R, Sasagawa T, Ninomiya I, Miwa K, Tanii H, Saijoh $\mathrm{K}$. Reduction in the local expression of complement component 6 (C6) and 7 (C7) mRNAs in oesophageal carcinoma. Eur J Cancer. 2001; 37: 1158-65.

19. Suryawanshi S, Huang X, Elishaev E, Budiu RA, Zhang L, Kim S, Donnellan N, Mantia-Smaldone G, Ma T, Tseng G, Lee T, Mansuria S, Edwards RP, et al. Complement pathway is frequently altered in endometriosis and endometriosisassociated ovarian cancer. Clin Cancer Res. 2014; 20: 616374. doi: 10.1158/1078-0432.CCR-14-1338.

20. Mamidi S, Hone S, Kirschfink M. The complement system in cancer: Ambivalence between tumour destruction and promotion. Immunobiology. 2015. doi: 10.1016/j. imbio.2015.11.008.

21. Zhou W. The new face of anaphylatoxins in immune regulation. Immunobiology. 2012; 217: 225-34. doi: 10.1016/j.imbio.2011.07.016.

22. Corrales L, Ajona D, Rafail S, Lasarte JJ, Riezu-Boj JI, Lambris JD, Rouzaut A, Pajares MJ, Montuenga LM, Pio R. Anaphylatoxin C5a creates a favorable microenvironment for lung cancer progression. J Immunol. 2012; 189: 467483. doi: 10.4049/jimmunol.1201654.

23. Shenhua X, Hanzhou M, Lijuan Q, Yongzhen S, Chihong Z, Xiaoshu H, Yongliang G, Shanzing D. Establishment and characterization of a model of highly metastasizing numan ovarian cancer transplanted into subcutis of the nude mice. 1995.

24. Shenhua X, Lijuan Q, Hanzhou N, Xinghao N, Chihong Z, Gu Z, Weifang D, Yongliang G. Establishment of a highly metastatic human ovarian cancer cell line (HO-8910PM) and its characterization. J Exp Clin Cancer Res. 1999; 18: 233-9.

25. Scully RE. (1999). Histological typing of ovarian tumours. (New York: Springer Berlin Heidelberg).

26. Shepherd JH. Revised FIGO staging for gynaecological cancer. Br J Obstet Gynaecol. 1989; 96: 889-92.

27. Beasley MB, Brambilla E, Travis WD. The 2004 World Health Organization classification of lung tumors. Seminars in Roentgenology. 2005; 40: 90-7. doi: 10.1053/j. ro.2005.01.001.

28. Goldstraw P. (2009). 7th Edition of TNM for Lung and Pleural Tumours. In: Goldstraw P, ed. Staging Manual in Thoracic Oncology. (Florida: Orange Park), pp. 57-65.

29. Szasz AM, Lanczky A, Nagy A, Forster S, Hark K, Green JE, Boussioutas A, Busuttil R, Szabo A, Gyorffy B. Crossvalidation of survival associated biomarkers in gastric cancer using transcriptomic data of 1,065 patients. Oncotarget. 2016; 7:49322-49333. doi: 10.18632/oncotarget.10337. 